\title{
Structural Characterization of Lab-Evolved Proteins Reveals Signature Sequences Required for High-Affinity Binding to HIV-1 TAR RNA
}

\author{
Sai Shashank Chavali, Ivan A. Belashov, Jermaine Jenkins and Joseph E. Wedekind* \\ Department of Biochemistry \& Biophysics, and Center for RNA Biology, University of Rochester \\ School of Medicine and Dentistry, Rochester NY 14642, USA.
}

*Corresponding author: e-mail: joseph.wedekind@rochester.edu

Innovative approaches are needed to create new therapeutics that target HIV, especially those that act on novel facets of the viral life cycle. The HIV-1 TAR (trans-activation response) RNA is a validated drug target that resists mutations to maintain interactions with the viral protein Tat and host pTEFb factors, giving rise to a protein-RNA complex that is essential for viral transcription. So far, TAR has evaded discovery of compounds with sufficient affinity and selectivity to warrant pharmaceutical development. To meet this challenge, we pursued a novel protein-evolution approach that yielded many high-affinity TAR Binding Proteins (TBPs) derived from the U1A spliceosomal protein. Here we describe the binding analysis of several TBPs, and a detailed structural analysis of the TBP6.7-TAR at $1.80 \AA$ resolution. TBP6.7 recognizes conserved guanines in the major groove using three arginines emanating from the lab-evolved $\beta 2-\beta 3$ loop. We further demonstrate the feasibility of reducing TBP6.7 to a short, stapled peptide that retains TAR binding and inhibits Tat-mediated transcription in HeLa lysate. We also present structural and binding analysis on additional TBPs $(6.3,6.6,6.9 \& 6.25)$, which reveals the importance of arginine distribution in the $\beta 2-\beta 3$ loop for TAR recognition. Mutagenesis and ITC experiments on indicate that T50 of the $\beta 2-\beta 3$ loop is crucial to stabilize productive arginine-backbone interactions, and that arginine can be accommodated only at certain positions in the $\beta 2-\beta 3$ loop for high-affinity TAR binding. This information is being used to optimize peptides that target HIV1 TAR to improve our understanding of protein-RNA molecular recognition. 